\title{
A NEW APPROACH TO PHOTON BEAM POSITION MONITORING AT ELETTRA
}

\author{
A. Galimberti ${ }^{1}$, C. J. Bocchetta, A. Gambitta, G. Paolucci, SINCROTRONE TRIESTE \\ G. Paolicelli, INFM UNITA' ROMA TRE \\ G. Stefani, UNIVERSITA' DI ROMA TRE AND INFM UNITA'ROMA TRE
}

\begin{abstract}
In the latest generation of SR sources, local bump orbit feedback systems are under development for improving the stability of the delivered radiation. Photon Beam Position Monitors (PBPM) are being built as detectors of beam movements and as references for feedback systems. A new generation of PBPM's for Undulator beamlines is being designed at ELETTRA. Detectors based on the use of electron energy analysers will drastically reduce the contamination of dipole magnet radiation on the PBPM to less than $0.1 \%$. A detector prototype has been designed and is currently under construction. A full description of the detector layout, its working principle and the expected behaviour obtained by computer simulations are presented.
\end{abstract}

\section{INTRODUCTION}

For the latest generation of SR sources, local bump orbit feedback systems are being developed for the improvement of the stability of the delivered radiation [1].

The conventional approach to a PBPM system for a high brightness beamline from an Insertion Device (ID) is to provide a pair of detectors based on four blades that intercept the fringes of the beam and photoemit electrons.

The positions of the beam centre in the vertical and horizontal planes and the angles of emission are then computed from the eight photocurrents measured. At ELETTRA, in all the front-ends of the ID beamlines, a PBPM system is installed [2]. Because of our storage ring configuration, a significant part of the radiation from the upstream and downstream dipoles is superimposed to the ID beamline radiation [3]. This fact inhibits the application of any feedback algorithm based on the PBPM measurements.

\section{DETECTOR LAYOUT}

Even if the peak intensity of the Undulator radiation is much higher than the Bending Magnet (BM) contribution, the integrated intensity seen by a conventional PBPM, can be comparable. A drastic reduction of the contribution of dipole magnet radiation down to less than $0.1 \%$ can be achieved by selecting the energy of the photoelectrons. The Undulator energy spectrum is peaked within a narrow bandwidth around a particular value, which changes with the $\mathrm{K}$ parameter and the harmonics. The energy range of the $\mathrm{BM}$ radiation is wide and goes from few eV to $20 \mathrm{KeV}$ with a photon flux at $2 \mathrm{GeV}$ of about $10^{13}$ [photons $/ \mathrm{s} / \mathrm{mrad}^{2} / 0.1 \% \mathrm{BW}$ ]. Furthermore, only the fringe fields of the dipole contribute to the contamination of the
PBPM, so the effective BM photon flux seen is expected to be some order of magnitude smaller.

On the other hand, the first harmonic photon flux of, for example, the Elliptical Wiggler (section 4.2) working in the Plane Undulator Mode at $\mathrm{K}_{1}=1.1$ is about $1 \times 10^{16}$ [photons $/ \mathrm{s} / \mathrm{mrad}^{2} / 0.1 \% \mathrm{BW}$ ] at a photon energy of $112 \mathrm{eV}$. Therefore, we have designed a new detector that selects the contribution to the spectrum at a given photon energy, with a bandwidth comparable to the Undulator linewidth. It will thus take advantage of the flux difference between $\mathrm{BM}$ and ID contribution at the positions of the harmonics.

A first prototype is under development and its layout is depicted in fig. 1. It is designed for position detection on a single plane and is composed of two parts: the photoemissive blades and a pair of electron energy analysers. The electron analysers are composed by an input lens and a hemispherical dispersing element. Each analyser is aligned with a blade and it collects the electrons, with an angular acceptance of a tenth of mradian, photoemitted by the blade.

The geometric position of the electrostatic lens and its polarisation ensure that each system (analyser \& blade) is independent of the other. Tuning the analyser at the proper energy, depending on the Undulator spectrum and the photoemission line of the blade, we are able to reject most of the BM contribution to the signal.

The detector is energy tuneable from few eV to some $\mathrm{keV}$. Therefore, it is suitable, for every Undulator device. Moreover it may be set easily and quickly for any working gap of the Undulator.

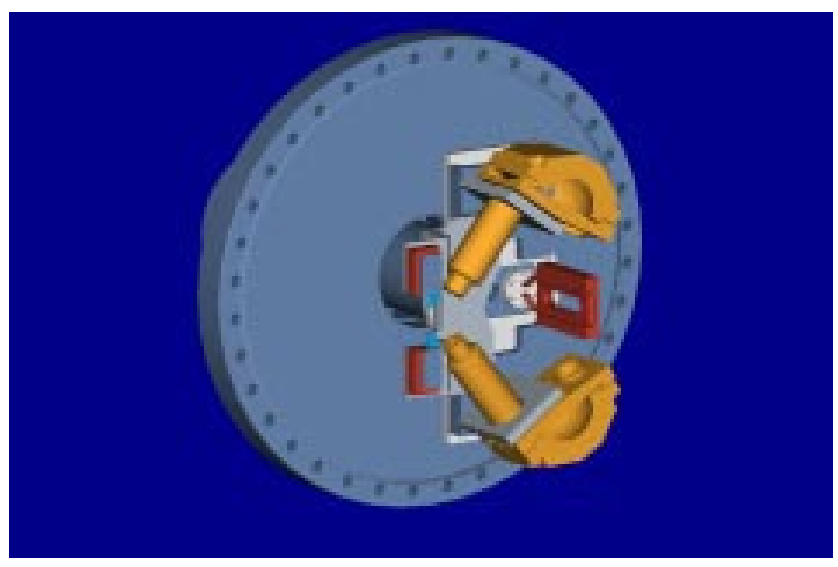

Figure 1: The NewPBPM prototype layout. The flange of the manipulator supports the detector. The main active elements are visible: the couple of blades and the hemispherical analysers that collect and filter the photoemitted electrons.

galimberti@elettra.trieste.it 


\section{DETECTOR WORKING PRINCIPLE}

\subsection{The Electron Analyser}

The new PBPM utilises the electrostatic hemispherical analyser [4] developed and used, by the Aloisa and GasPhase beamlines at ELETTRA; to perform angle resolved time coincidence photoemission experiments. This analyser is characterised by a compact hemispherical dispersing element $\left(\mathrm{R}_{0}=33 \mathrm{~mm}\right)$, an electrostatic lens with a cylindrical symmetry formed by four elements and a system of selectable input and exit slits. All these features have been fully exploited on the experimental end stations at ELETTRA.

The dispersing element, composed of a pair of concentric hemispheres with fixed input and exit slits, selects electrons of a particular energy $E_{p}$ proportional to the voltage difference between the hemispheres $\left(\mathrm{E}_{\mathrm{p}}=\mathrm{k}_{1} \Delta \mathrm{V}\right)$. It is characterised by a constant relative energy resolution $\Delta \mathrm{E} / \mathrm{E}=\mathrm{k}_{2}$ [5]. The dimension of the internal and external hemisphere determines the constant $\mathrm{k}_{1}$, which is fixed for a given spectrometer. The constant $\mathrm{k}_{2}$ is inversely proportional to the mean radius $\mathrm{R}_{0}$ and directly proportional (in first order approximation) to the slit width so the relative resolution can be varied by changing the settings of the slits set when the experimental chamber is in air. Energy spectra can be performed in two ways: changing the kinetic energy of the analysed particle to match the fixed value selected by a constant applied voltage on the hemispherical sector or moving the applied potential on the hemisphere according to proportional factor $\mathrm{k}_{1}$. With the help of an input electrostatic lens, we can work in both conditions preserving optimised efficiency. The first operation mode is used to keep constant the energy resolution. The lens focuses electrons from blade to the hemisphere and changes, in a controlled way, their kinetic energy $E$ to a fixed value to reach constant energy resolution $\left(\Delta \mathrm{E}=\mathrm{k}_{2} \mathrm{E}_{\mathrm{p}}\right)$. The lens transmission changes during the energy scan because angular magnification and linear magnification vary $\left(M_{L} \cdot M_{\alpha}=\sqrt{\frac{E}{E_{p}}}\right)$. The second operation mode is characterised by a constant lens transmission because the lens is used just to focus electrons and eventually to change their energy by a fixed proportional factor $\left(\frac{E}{E_{p}}=\right.$ Retarding Ratio $\left.=\mathrm{R}\right)$. Since in the present application, the essential point is not energy resolution but a controlled and possibly, constant transmission of the spectrometer for different analysed energy ranges, we plan to work in the second operation mode called Constant Relative Resolution. The intrinsic relative resolution $\mathrm{k}_{2}$ has been estimated by computer simulation considering second order angular terms. Imposing a slit width of $2 \mathrm{~mm}$, it results to be of the order of $3.5^{*} 10^{-2}$. So it is comparable to the relative bandwidth of Undulator harmonics $\Delta \mathrm{E} / \mathrm{E}=(1$ to5 $) 10^{-2}$. To achieve a fine tuning of the overall analyser relative bandwidth with respect to the Undulator line shape we have optimised the optical system for a Retarding Ratio ranging from 1 to 5 .
As an example we may consider a lens system working at $R=2$; the overall behaviour results:

$$
\Delta E / E=\left(\Delta E / E_{p} R\right)=\left(\Delta E / E_{p}\right) \frac{1}{R}=\frac{3.5}{2} \cdot 10^{-2} \approx 2 \cdot 10^{-2}
$$

\subsection{The Data Acquisition System}

The instrumentation set-up of this detector is more complex than for a standard PBPM. Firstly, each electrode of the electron analysers and their electrostatic lenses is controlled by a set of low ripple programmable HV power supplies. Secondly, at each electron analyser exit an electron multiplier, that increases the signal level, has to be biased to some $\mathrm{kV}$ for its proper functioning. Two different ways of data acquisition are needed: counting mode and current mode. Each mode has a dedicated electronics: the former detects the fast pulse train coming out from the analyser and counts them. The latter integrates, with a configurable time constant, the signal. Then in both cases the data is processed in order to find the vertical beam centre.

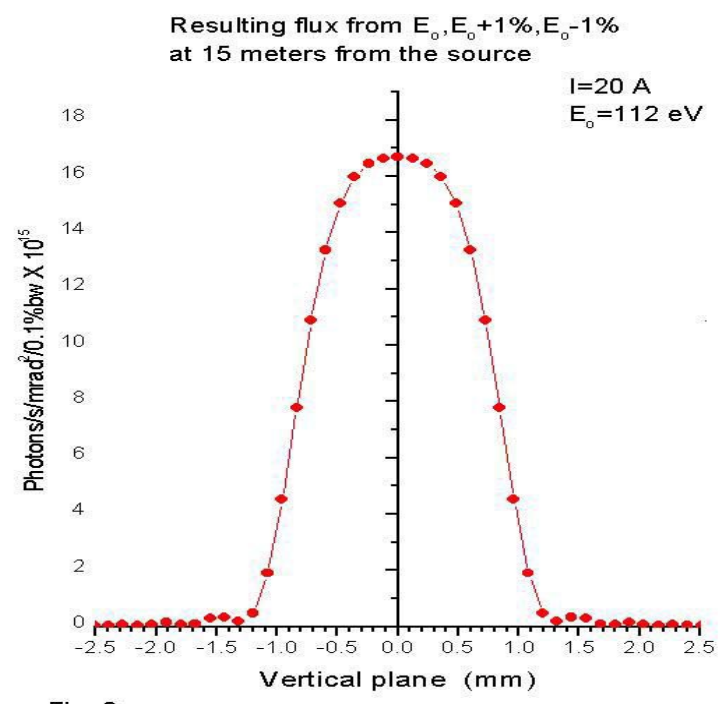

Fig. 2

Figure 2: Photon flux spatial distribution for the insertion device ID4 tuned at $112 \mathrm{eV}$ within an energy bandwidth of $\pm 1 \%$ (@15m from the source)

Moreover, the two photocurrents of the blades are also acquired for alignment purposes. The acquisition in current mode is performed by custom floating picoammeters to decouple the biasing HV.

\section{SYSTEM SIMULATION}

The behaviour of the analyser has been studied by an electron ray-tracing simulation [6]. In particular we have made an effort to evaluate the spectrometer Field of View (or better the spatial transmission function at fixed analysed energy). In fact, the convolution of this curve with the photon flux spatial distribution (Fig. 2) determines the sensitivity of our system to beam motion. The result of the simulation is shown in fig. 3 for an 
analyser configuration characterised by $\Delta \mathrm{E} / \mathrm{E}=2 \%$. A grid of emission points, placed at the blade surface, has been utilised $(0.1 \times 0.5 \mathrm{~mm}$ spacing). Each point emits electrons in two orthogonal planes both perpendicular to the blade surface with a symmetric emission angle of $\pm 6^{0}$ (the lens geometrical acceptance angle). All the electrons have the same kinetic energy and lens and hemisphere voltages are tuned for this energy. The green colour scale represents the fraction of trajectories that reaches the exit slit of the hemisphere.

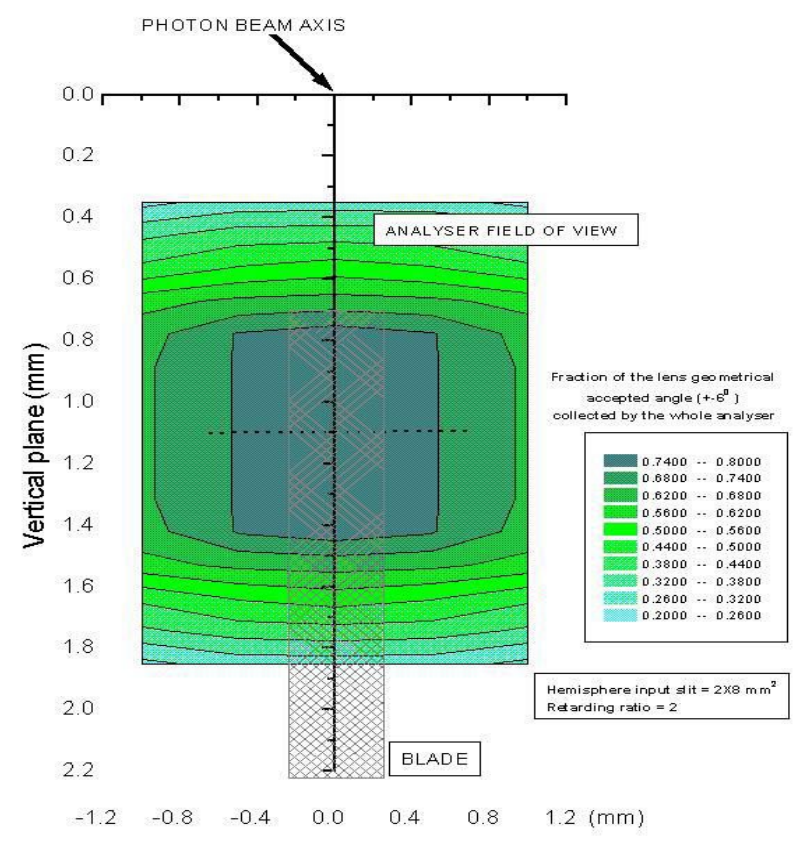

Figure 3: A simulation of the spectrometer transmission function with a $\Delta \mathrm{E} / \mathrm{E}=2 \%$.

The electron beam orbit stability has to achieve $0.1 \%$ intensity constancy of the delivered radiation. Therefore, the Synchrotron light sources have stability goals from $10 \%$ to $1 \%$ of the photon beam size and divergence [7]. At $15 \mathrm{~m}$ from the source it means a detector sensitivity of about $10 \mu \mathrm{m}$.

In order to estimate the sensitivity we consider that measured signal will be proportional to the convolution of spectrometer transmission function (fig.3) with the photon beam distribution (fig.2). We have calculated this curve along the vertical plane for a centred beam and for a $10 \mu \mathrm{m}$ beam displacement. The result indicates a relative difference between the two signals of the order of $4 \%$. The estimated current signal at the exit of the analyser is about $\ln \mathrm{A}$. So a current measurement with the accuracy of $10 \mathrm{pA}$ is able to achieve a beam position sensitivity better than $10 \mu \mathrm{m}$. The electronics allow an accuracy of $1 \mathrm{pA}$ and so it would be possible to reach a sensitivity of $1 \mu \mathrm{m}$.

\section{THE UHV CHAMBER AND TEST MEASUREMENTS}

An UHV test chamber for PBPM testing will be placed along the Circular Polarisation Beamline immediately after the front-end and before any optical element. A white beam is available at this location 15 meters from the source. An X-Z manipulator will provide the translations of the detector on an orthogonal plane with respect to the beam. In addition each pair of blade and analyser is mounted on a movable support that allows change the distance between the two blades. Therefore it is possible to change the working position of the detector with respect to the photon beam. The chamber has to be built as a compact experimental chamber with particular attention paid to the magnetic shielding.

\section{PRESENT STATUS}

Currently all the mechanical components are under construction. Delivery is planned for this summer. The control system and the instrumentation have been designed and are in construction. The first measurements with the detector are planned for the end of the year.

\section{CONCLUSIONS}

A new possible solution to photon beam position monitoring for Undulator beamlines has been presented. The simulations confirm that it is possible to obtain good sensitivity and to reduce drastically the contamination from BM radiation. The design allows a great flexibility and a large operative range. The measures will confirm if it is also completely compatible with the strictly requirements for the orbit stability feedback controls.

\section{ACKNOWLEDGEMENTS}

The authors would like to thank B. Diviacco for his scientific suggestions and C. Fava for his technical skill and friendly collaboration.

\section{REFERENCES}

[1] C. J. Bocchetta et al.: 'The Design of the ELETTRA Fast Local Feedback System', Proc. 17th PAC, Vancouver, Canada, 1997.

[2] A. Galimberti et al.: 'Photon Beam Position Monitors suitable for a Local Feedback System at ELETTRA', Proc. 5th EPAC, Sitges, Spain, 1996.

[3] A. Galimberti and C. Scafuri: 'A Preliminary Study on High Precision Photon Beam Position Monitor Design for Local Feedback Systems', Proc. 6th EPAC, Stockholm, Sweden, 1998.

[4] G. Stefani, M.V. Marabello: 'The Electron Spectrometer for the ALOISA Beamline', INFM, Internal report, 1991.

[5] E.H.A. Granneman and M.J. Van der Wiel: 'Transport, Dispersion and Detection of Electrons, Ions and Neutrals' in Handbook of Synchrotron radiation, Vol. 1, Ed.by E.E. Koch North Holland 1983.

[6] SIMION 3D code by David A. Dahl, Idaho National Engineering Laboratory, 1995.

[7] H. Winick: 'Synchrotron Radiation Sources', World Scientific, 1994. 